\title{
ANALYSIS OF THE PENALTY CORNER ATTACK STRATEGY IN INTERNATIONAL WOMEN'S HOCKEY: 2016 CHAMPIONS TROPHY AND 2016 OLYMPIC GAMES IN RIO DE JANEIRO
}

original paper

( ) University School of Physical Education in Wroclaw

DOI: https://doi.org/10.5114/hm.2018.76083

\author{
JEHEON MOON $^{1,2}$, JONGCHUL PARK ${ }^{2}$, KEONWOOK KANG $^{3}$ \\ ${ }^{1}$ Department of Human Performance and Health Education, Western Michigan University, Kalamazoo, USA \\ ${ }^{2}$ Department of Sports Science, Korea Institute of Sport Science, Seoul, Republic of Korea \\ ${ }^{3}$ Department of Physical Education, Korea National Sport University, Seoul, Republic of Korea
}

\section{ABSTRACT}

Purpose. The purpose of the study was to analyse the differences in various penalty corner attack tactics observed in recent international women's hockey matches, including the 2016 Champions Trophy (CT) and 2016 Olympic Games in Rio de Janeiro (OG), and to propose factors that lead to a successful penalty corner attack.

Methods. The study examined 204 cases of penalty corner attack (CT: 111, OG: 93) that occurred in 29 games of international women's hockey matches (CT: 18, OG: 11); these were analysed with the chi-square test and binary logistic regression analysis. Results. Differences were observed with respect to the set play position $\left(\chi^{2}=19.091, p=0.002\right)$, shooting point $\left(\chi^{2}=5.355\right.$, $p=0.021)$, and shooting direction $\left(\chi^{2}=22.831, p=0.004\right)$. The binary logistic regression, used to determine factors influencing the score, revealed that the chance of scoring increased with the use of set play $(p=0.015)$. However, the chance of failing to score is higher if the shooter makes a pass instead of a direct shot $(p=0.023)$.

Conclusions. When analysing factors that affect penalty corner goals in women's hockey, a higher success rate for goalscoring was seen with set plays. However, the failure rate was higher when the shooter used a passing technique instead of direct shooting. Thus, a strategy focusing on a constructed set play rather than passing techniques around stoppers should be established to increase the success rate of goals.

Key words: field hockey, tactical skills, set play

\section{Introduction}

Elite team sports have adopted performance profiling, which gathers physiological, technical, and tactical information needed to win the game [1]. In terms of exercise physiology, heart rate and sessional ratings of perceived exertion were analysed to evaluate exercise intensity of a periodic training program [2, 3], and technical indicators refer to extrinsic factors such as shooting motion analysis [4-6]. Information on strategy and tactics is crucial in situations with restricted competition, such as the hockey penalty corner in international competitions, with minimal differences in performance.

Studies analysing past hockey matches have recorded images to assess changes in movement patterns. The results from these studies show that the stride length in running increases in response to the decrease in intensity with the progression of the match [7-9]. Since the development of the Global Positioning System (GPS), sports scientists and conditioning coaches have been able to obtain more specific and quantitative information, such as sprint time, distances, and velocities performed by players during training period as well as the competitions. As a result, it was observed that while the athletes moved on an average of $4.8 \mathrm{~km}$ per game, the defenders covered a greater total distance and the midfielders performed a greater moderate intensity distance [10]. While the forwards performed the highest level of moderate- and high-intensity running, the time of the event was the shortest $[10,11]$.

Correspondence address: Jongchul Park, Department of Sports Science, Korea Institute of Sport Science,

727 Hwarang-lo Nowon-gu, Seoul, 139-242, Republic of Korea, e-mail: mori@kspo.or.k

Received: November 17, 2017

Accepted for publication: April 23, 2018

Citation: Moon J, Park J, Kang K. Analysis of the penalty corner attack strategy in international women's hockey: 2016 Champions Trophy and 2016 Olympic Games in Rio de Janeiro. Hum Mov. 2018;19(3):82-87; doi: https://doi.org/10.5114/ hm.2018.76083. 
Such information is useful in substituting players during a match and establishing the intensity of training; however, its relevance to the strategy of the penalty corner situation is minimal.

Penalty corners typically account for approximately $20-30 \%$ of goals scored in hockey [12]. As this is the most effective method of attack that could change the flow of the game in a losing situation, an attack strategy is needed to increase the rate of success $[12,13]$. Of these, scoring a goal by direct drag-flicks had the highest ratio of $67 \%$. In addition, other tactics have demonstrated that the goalkeeper needs to rush to the edge of the circle for a successful defence. In particular, in a penalty corner situation in which players move under a certain operation, the type and direction of shooting raise the probability of scoring and the position of defence with regard to the goalkeeper can be extremely useful information [14]. Moreover, detailed information on how set plays are being conducted during penalty corner attacks, along with the number of stoppers being utilized, can provide an insight regarding game strategies and tactics for women's hockey penalty corners.

In our study, we particularly wanted to perform match analysis of high-level competitive tournaments such as the 2016 Champions Trophy (CT) and 2016 Olympic Games in Rio de Janeiro (OG), so that it can provide us with explanations regarding the factors that contributed to the successes and failures in these recent tournaments. Therefore, the purpose of our study was to perform a logistic regression analysis of attacking strategies employed by teams in a penalty corner during recent women's hockey matches and to extract factors that increase the success rate in penalty corner goals.

\section{Material and methods}

\section{Sample}

The sample data used for analysis in the present study include 204 cases of penalty corner situations (CT: 111, OG: 93) that occurred in 29 international women's hockey matches (CT: 18, OG: 11). As the countries that participated in both CT and OG competitions comprise 6 teams (Argentina, Australia, England, Netherlands, New Zealand, and the United States) with world-class performances, an analysis of their performances would provide extremely useful data.

\section{Development of the system}

Through cooperation among researchers, national women's hockey leaders, and performance analysts, a system was developed to enter the main factors for penalty corners in real time. Factors for analysis included the number of stoppers, use of pass, use of set plays, set play position, shooting point, technique, and direction [14]. These factors were classified as pass (number of stoppers, use of pass), set play (use of set play, set play position), and shooting (shooting point, technique, direction). Such classification is explained below:

1. Number of stoppers: either 1 or 2 players behave as stoppers to confuse the position of the shooter.

2. Use of pass: shot pass by the stopper after receiving the ball by either direct shooting or passing the ball to a shooter nearby.

3. Use of set play: set play occurs when the shooter does not attempt shooting but makes a strong pass to a fellow shooter who is rushing to yield a shooting.

4. Set play position: 6 types of position employed by players other than pusher, stopper, and shooter who participate in attack (L0-R1, L0-R2, L1-R1, L1-R2, L1-R0, L2-R0), with L as left, $\mathrm{R}$ as right and figures as number of players.

5. Shooting point: the point of shooting (either left or right side of the edge of the circle) relative to the goal performed by the shooter.

6. Shooting technique: 3 kinds of techniques used by the shooter while shooting (flick - push without swing, hit - hitting with swing, drag flick - slide with shooting).

7. Shooting direction: 9 types of shooting direction relative to the goal (Figure 1).

Video data collection was performed in the shooting area within the stadium to record every movement of the players easily. Two video cameras (Sony, HXRNX70N, Japan) were used in image recording, and one

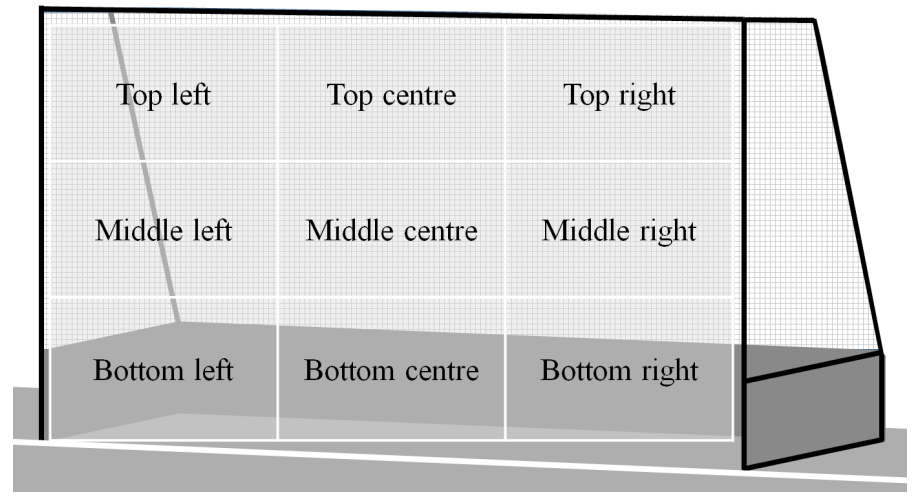

Figure 1. Types of shooting direction relative to the goal 


\section{HUMAN MOVEMENT}

J. Moon, J. Park, K. Kang, Penalty corner strategy in women’s hockey

of them was used as a dedicated penalty corner camera. The recording speed of all cameras was set to 60 frames/s. Data coding was performed by image analysis experts during the game in real time. For software, Sportscode Elite v. 10.3.28 (Sportstec, New South Wales, Australia) was used.

\section{Data reliability}

Data recorded by image analysis experts were reviewed by 2 national women's hockey leaders ( 1 of them was a goalkeeper coach) to ensure the internal validity of performance profiling. The technical error of measurement is paired with the change in mean to evaluate the reliability of results in terms of the level of agreement between initial tests and any subsequent retests [15]. The level of agreement between tests and retests was determined via Cohen's kappa statistics [16]. In this study, Cohen's kappa value of 0.91 (range: 0.870.94) was considered as an acceptable reliability level.

\section{Statistical analysis}

All verified data for each competition were exported to a Microsoft Excel spreadsheet and organized. A chisquare test was performed to verify differences among competitions. The type of competition (CT, OG) was listed in horizontal lines, and 7 types of dependent variables (i.e., the types mentioned above) were listed in columns [17]. Furthermore, binary logistic regression analysis was used to identify factors that affect the penalty corner goals $[13,17]$. In this analysis, the independent variable was the outcome of the goal, and the covariates were the 7 types of attack techniques. Collinearity diagnostics revealed that the relevance among independent variables was not high (minimum tolerance: 0.195 , maximum variance inflation factor: 5.124). For these results, a forced entry method was used. Other potential procedures, such as a stepwise procedure, lacked satisfactory volume to provide sufficiently valid results [18]. The statistical analysis was performed with the SPSS for Windows v. 18.0 (SPSS, Inc., Chicago, USA) and the statistical significance level was set at $p<0.05$.

\section{Ethical approval}

The research has been complied with all the relevant national regulations and institutional policies, has followed the tenets of the Declaration of Helsinki, and has been approved by the institutional ethics committee of the Korea Institute of Sport Science.

\section{Results}

In this study, the analysis was performed with 204 cases of penalty corner techniques (CT: 111, OG: 93) that occurred in the total of 29 games (CT: 18, OG: 11). In CT, 24 cases (21.6\%) led to a goal. In OG, 17 cases

Table 1. Results of the chi-square test on the number of stoppers, use of the pass technique, use of set play, and set play position in relation to the type of match

\begin{tabular}{|c|c|c|c|c|c|}
\hline Variables & & $\mathrm{CT}$ & OG & Total & $\chi^{2}$ \\
\hline \multirow{3}{*}{ Number of stoppers } & 1 & $15(7.4 \%)$ & $12(5.9 \%)$ & $27(13.2 \%)$ & \multirow{3}{*}{$\begin{array}{c}0.016 \\
(p=0.898)\end{array}$} \\
\hline & 2 & $96(47.1 \%)$ & $81(39.7 \%)$ & $177(86.8 \%)$ & \\
\hline & Total & $111(54.4 \%)$ & $93(45.6 \%)$ & $204(100 \%)$ & \\
\hline \multirow{3}{*}{ Use of the pass } & Yes & $43(21.1 \%)$ & $43(21.1 \%)$ & $89(43.6 \%)$ & \multirow{3}{*}{$\begin{array}{c}2.366 \\
(p=0.124)\end{array}$} \\
\hline & No & $68(33.3 \%)$ & $47(23.0 \%)$ & 115 (56.4\%) & \\
\hline & Total & $111(54.4 \%)$ & $93(45.6 \%)$ & $204(100 \%)$ & \\
\hline \multirow{3}{*}{ Use of set play } & Yes & $24(11.8 \%)$ & $13(6.4 \%)$ & $37(18.1 \%)$ & \multirow{3}{*}{$\begin{array}{c}1.991 \\
(p=0.158)\end{array}$} \\
\hline & No & $87(42.6 \%)$ & $80(39.2 \%)$ & $167(81.9 \%)$ & \\
\hline & Total & $111(54.4 \%)$ & $93(45.6 \%)$ & $204(100 \%)$ & \\
\hline \multirow{7}{*}{ Set play position } & L0-R1 & $40(19.7 \%)$ & $16(7.8 \%)$ & $56(27.5 \%)$ & \multirow{7}{*}{$\begin{array}{c}19.091 \\
(p=0.002)\end{array}$} \\
\hline & L0-R1 & $59(28.9 \%)$ & $65(31.9 \%)$ & $124(60.8 \%)$ & \\
\hline & L1-R0 & $2(1.0 \%)$ & $2(1.0 \%)$ & $4(2.0 \%)$ & \\
\hline & L1-R1 & $1(0.5 \%)$ & $7(3.4 \%)$ & $8(3.9 \%)$ & \\
\hline & L1-R2 & $9(4.4 \%)$ & $2(1.0 \%)$ & $11(5.4 \%)$ & \\
\hline & L2-R0 & $0(0 \%)$ & $1(0.5 \%)$ & $1(0.5 \%)$ & \\
\hline & Total & $111(54.4 \%)$ & $93(45.6 \%)$ & $204(100 \%)$ & \\
\hline
\end{tabular}

CT - 2016 Champions Trophy, OG - 2016 Olympic Games in Rio de Janeiro 
Table 2. Results of the chi-square test on shooting points, techniques, and directions in relation to the type of match

\begin{tabular}{llcccc}
\hline Variables & & CT & OG & Total & $\chi^{2}$ \\
\hline \multirow{5}{*}{ Shooting point } & Right & $61(29.9 \%)$ & $36(17.6 \%)$ & $97(47.5 \%)$ & 5.355 \\
& Left & $50(24.5 \%)$ & $57(27.9 \%)$ & $107(52.5 \%)$ & $(p=0.021)$ \\
& Total & $111(54.4 \%)$ & $93(45.6 \%)$ & $204(100 \%)$ & \\
& Flick & $81(40.3 \%)$ & $69(34.3 \%)$ & $150(74.6 \%)$ & \\
Shooting technique & Hit & $8(4.0 \%)$ & $8(4.0 \%)$ & $16(8.0 \%)$ & 1.092 \\
& Drag flick & $22(10.9 \%)$ & $13(6.5 \%)$ & $35(17.4 \%)$ & $(p=0.579)$ \\
& Total & $111(55.2 \%)$ & $90(44.8 \%)$ & $201(100 \%)$ & \\
\hline & Bottom left & $24(23.5 \%)$ & $12(11.8 \%)$ & $36(35.3 \%)$ & \\
& Bottom centre & $6(5.9 \%)$ & $9(8.8 \%)$ & $15(14.7 \%)$ & \\
& Bottom right & $11(10.8 \%)$ & $3(2.9 \%)$ & $14(13.7 \%)$ & \\
& Middle left & $0(0 \%)$ & $6(5.9 \%)$ & $6(5.9 \%)$ & 22.831 \\
& Middle centre & $4(3.9 \%)$ & $4(3.9 \%)$ & $8(7.8 \%)$ & $(p=0.004)$ \\
& Middle right & $12(11.8 \%)$ & $2(2.0 \%)$ & $14(13.7 \%)$ & \\
& Top left & $2(2.0 \%)$ & $0(0 \%)$ & $2(2.0 \%)$ & \\
& Top centre & $0(0 \%)$ & $2(2.0 \%)$ & $2(2.0 \%)$ & \\
& Top right & $3(2.9 \%)$ & $2(2.0 \%)$ & $5(4.9 \%)$ & \\
& Total & $62(60.8 \%)$ & $62(60.8 \%)$ & $102(100 \%)$ & \\
& & & & \\
\end{tabular}

CT - 2016 Champions Trophy, OG - 2016 Olympic Games in Rio de Janeiro

$(18.3 \%)$ led to a goal. The result of the chi-square test on the number of stoppers, the use of the pass technique, and the use of set play in relation to the match type did not demonstrate a statistical significance (Table 1). The results of the chi-square test on the type of set play position in relation to the match showed a statistical significance $\left(\chi^{2}=19.091, p=0.002\right)$. In CT, positions L0-R1 and L0-R2 were used almost equally in 40 cases $(19.7 \%)$ and 59 cases $(28.9 \%)$, respectively. In OG, position L0-R2 was applied in 65 cases (31.9\%), significantly more often than other position types.

The chi-square test analysis on shooting points in relation to the type of match revealed a statistically significant difference (Table 2). In CT, there was a higher frequency of shooting from the right, with 61 cases (29.9\%); in OG, a higher frequency was observed of the left shooting point, with 57 cases $(27.9 \%)\left(\chi^{2}=\right.$ $5.355, p=0.021)$. On the basis of the chi-square analysis, there was no statistical difference between the shooting technique and type of match. The results of the chi-square test on shooting direction in relation to the match type showed a statistically significant difference. In CT, the bottom left and middle right shooting directions were used most frequently, in 24 cases (23.5\%) and 12 cases (11.8\%), respectively; in OG, bottom left and bottom centre were used more fre-

Table 3. Results of logistic regression analysis on covariates

\begin{tabular}{|c|c|c|c|c|c|c|c|c|}
\hline \multirow{2}{*}{ Covariates } & & \multirow{2}{*}{$B$} & \multirow{2}{*}{$S E$} & \multirow{2}{*}{ Wald } & \multirow{2}{*}{$E X P(B)$} & \multirow{2}{*}{$p$} & \multicolumn{2}{|c|}{$95 \%$ CI for $\operatorname{EXP}(B)$} \\
\hline & & & & & & & Lower & Upper \\
\hline \multirow{2}{*}{ Pass } & Number of stoppers & 0.757 & 0.901 & 0.706 & 2.133 & 0.401 & 0.365 & 12.477 \\
\hline & Use of pass & -2.336 & 1.029 & 5.151 & 0.097 & 0.023 & 0.013 & 0.727 \\
\hline \multirow{2}{*}{ Set play } & Use of set play & 1.625 & 0.671 & 5.870 & 5.081 & 0.015 & 1.364 & 18.924 \\
\hline & Set play position & 0.318 & 0.301 & 1.121 & 1.375 & 0.290 & 0.763 & 2.479 \\
\hline \multirow{4}{*}{ Shooting } & Shooting point & 1.826 & 1.020 & 3.208 & 6.208 & 0.073 & 0.842 & 45.791 \\
\hline & Shooting technique & 0.157 & 0.403 & 0.151 & 1.170 & 0.697 & 0.531 & 2.576 \\
\hline & Shooting direction & 0.074 & 0.101 & 0.541 & 1.077 & 0.462 & 0.884 & 1.312 \\
\hline & Constant & -6.195 & 3.283 & 3.560 & 0.002 & 0.059 & & \\
\hline
\end{tabular}

$B$ - winning probability, positive values indicate a positive relationship, negative values indicate a negative correlation, $S E$ - standard error, Wald - the explanatory power of each dependent variable, $E X P(B)$ - odds ratio, indicating an increase in the winning probability, CI - confidence interval 
Table 4. The predictive accuracy of the logistic regression model

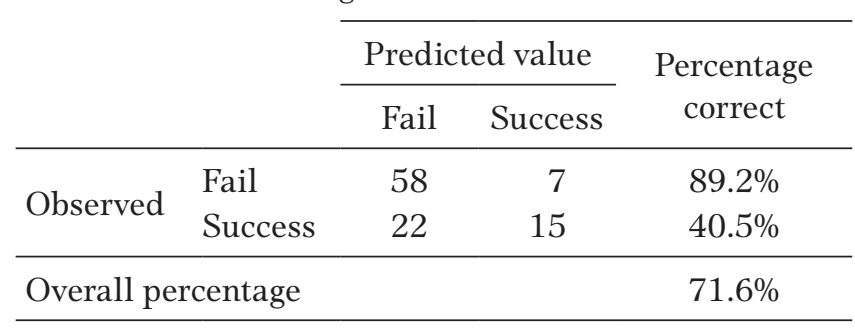

quently, with 12 cases $(11.8 \%)$ and 9 cases $(8.8 \%)$, respectively $\left(\chi^{2}=22.831, p=0.004\right)$.

The results of logistic regression analysis on covariates are presented in Table 3 . The use of pass had an inverse correlation with the score $(\mathrm{B}=-2.336)$, and the likelihood of failing to score was shown to increase 0.097 times ( $p=0.023)$. However, the use of set plays had a positive correlation with the score $(B=1.625)$, and the likelihood of succeeding to score was shown to increase 5.081 times $(p=0.015)$.

The predictive accuracy of the logistic regression model is presented in Table 4. For failures, 58 goals were predicted as a failure whereas 7 goals were predicted as a success, resulting in an accuracy of $89.2 \%$. Regarding the successes, 22 goals were predicted as a fail whereas 15 goals were predicted as a success, leading to an accuracy of $40.5 \%$. The overall accuracy reported by the regression equation was $71.6 \%$.

\section{Discussion}

The present study analysed the difference in penalty corner attack strategies used in recently held women's hockey competitions. In CT, 24 of 111 cases $(21.6 \%)$ of penalty corner attacks resulted in a goal. In OG, 17 of 93 cases (18.3\%) of penalty corner attacks resulted in a goal. Vinson et al. [12] reported that the success rate of penalty corner attacks in indoor hockey was $22.6 \%$, which is similar to the result reported in the present study.

On the analysis of the differences between $\mathrm{CT}$ and OG, both matches used the flick shooting technique the most (CT: $40.3 \%$, OG: 34.3\%). Concerning the shooting direction, the bottom had the highest rate (CT: $40.2 \%$, OG: $23.53 \%$ ), with most to the bottom left (CT: $23.5 \%$, OG: $11.8 \%)$. It was reported that the penalty corner shooter in women's hockey tended to use the strategy of flick shooting in the bottom left direction in relation to the goal $[19,20]$. This result was different in male players, who preferred the fast shooting technique [14, 21]. Shooting point from the left side of the goalie was used more frequently in OG than in
CT $\left(\chi^{2}=5.355, p=0.021\right)$. No significant difference between the 2 match types was observed in the use of direct shooting, use of set play, or the number of stoppers. A significant difference was noted with the set play position. In CT, positions L0-R1 and L0-R2 were used almost equally, whereas position L0-R2 was applied more frequently in OG $\left(\chi^{2}=19.091, p=0.002\right)$. The success rate of a goal may be attributable to this difference.

On binary logistic regression analysis, performed to identify factors affecting penalty corner scores, the likelihood of scoring a goal when using set play was 5.081 times higher $(p=0.015)$. The set play of both teams in CT and OG revealed a focus on having a set play position to the right (L0-R1 or L0-R2). Therefore, to score a goal from the penalty corner, various set play strategies should be developed, and players with good ball contact and stick skills should be positioned to rush from the back. Another factor with statistical significance was the use of the pass. There was an increase in the likelihood of failing to score, which was 0.097 times higher when the shooter passed instead of directly shooting $(p=0.023)$. Hence, the strategy to directly shoot rather than passing appears to result in a higher success rate in penalty corner attacks. According to the analysis of the German team's penalty corner cases in the 2010 Hockey World Cup, 16.7\% used the passing strategy, and $83.3 \%$ used the direct shooting strategy [13]. However, in women's hockey penalty corner cases, scoring a goal through deflection had a higher success rate than scoring through direct shooting $[14,19]$. Thus, a strategy focusing on a set play that places shooters with strong flick shooting skills is needed to confuse the opposing team's defensive tactics and succeed in a goal.

\section{Conclusions}

On the basis of the analysis of the differences in CT and OG, the frequency of placing 2 stoppers was higher in CT. With the set play position, CT cases more frequently placed 1 or 2 attackers on the right, whereas 2 attackers were often placed on the right in OG cases. A higher frequency of shooting points to the right was observed in CT, but more shooting points to the left were observed in OG. Concerning the shooting direction, the frequency of shooting toward the bottom left and middle right direction was higher in CT, and the frequency of shooting toward the bottom left and bottom centre was higher in OG. On the basis of binary logistic regression analysing factors that affect penalty corner goals, a higher success rate for goals was seen with set plays. However, the failure rate was higher 
when the shooter used the passing technique instead of direct shooting. Thus, a strategy focusing on constructed set plays rather than passing techniques around stoppers should be established to increase the success rate of goals. A set play that places shooters with strong flick shooting skills is needed to confuse the opposing team's defensive strategies. The present study depicted strategies with a high success rate of goal in the women's hockey penalty corner situation; however, it did not analyse defence strategies. Therefore, further research is needed to identify the ideal defence position and the role of the goalkeeper.

\section{Acknowledgments}

The authors acknowledge the Korea Institute of Sport Science for their financial support for this study.

\section{Disclosure statement}

No author has any financial interest or received any financial benefit from this research.

\section{Conflict of interest}

The authors state no conflict of interest.

\section{References}

1. O’Donoghue P. Sports performance profiling. In: McGarry T, O’Donoghue P, Sampaio J (ed.), Routledge handbook of sports performance analysis. London: Routledge; 2013; 127-139.

2. Perrotta AS, Held NJ, Warburton DE. Examination of internal training load parameters during the selection, preparation and competition phases of a mesocycle in elite field hockey players. Int J Perform Anal Sport. 2017; 17(5):813-821;doi:10.1080/24748668.2017.1402284.

3. Perrotta AS, Taunton JE, Koehle MS, White MD, Warburton DE. Monitoring the prescribed and experienced heart rate derived training loads in elite field hockey players. J Strength Cond Res. 2018; advance online publication; doi: 10.1519/JSC.0000000000002474.

4. De Baranda PS, Lopez-Riquelme D. Analysis of corner kicks in relation to match status in the 2006 World Cup. Eur J Sport Sci. 2012;12(2):121-129; doi: 10.1080/ 17461391.2010.551418.

5. Ibrahim R, Faber GS, Kingma I, van Dieën JH. Kinematic analysis of the drag flick in field hockey. Sports Biomech. 2016;16(1):45-57; doi: 10.1080/14763141. 2016.1182207.

6. Willmott AP, Dapena J. The planarity of the stickface motion in the field hockey hit. J Sports Sci. 2012;30(4): 369-377; doi: 10.1080/02640414.2011.642807.

7. MacLeod H, Bussell C, Sunderland C. Time-motion analysis of elite women's field hockey, with particular reference to maximum intensity movement patterns. Int J Perform Anal Sport. 2007;7(2):1-12; doi: 10.1080/ 24748668.2007.11868392.
8. Spencer M, Lawrence S, Rechichi C, Bishop D, Dawson B, Goodman C. Time-motion analysis of elite field hockey, with special reference to repeated-sprint activity. J Sports Sci. 2004;22(9):843-850; doi: 10.1080/02640 410410001716715.

9. Spencer M, Rechichi C, Lawrence S, Dawson B, Bishop D, Goodman C. Time-motion analysis of elite field hockey during several games in succession: a tournament scenario. J Sci Med Sport. 2005;8(4):382-391; doi: 10.1016/S1440-2440(05)80053-2.

10. McGuinness A, Malone S, Hughes B, Collins K. The physical activity and physiological profiles of elite international female field hockey players across the quarters of competitive match-play. J Strength Cond Res. 2018; advance online publication; doi: 10.1519/JSC. 0000000000002483.

11. Macutkiewicz D, Sunderland C. The use of GPS to evaluate activity profiles of elite women hockey players during match-play. J Sports Sci. 2011;29(9):967-973; doi: 10.1080/02640414.2011.570774.

12. Vinson D, Padley S, Croad A, Jeffreys M, Brady A, James D. Penalty corner routines in elite women's indoor field hockey: prediction of outcomes based on tactical decisions. J Sports Sci. 2013;31(8):887-893; doi: 10.1080/ 02640414.2012 .757341$.

13. Amjad I, Hussain I, Asadullah M. Comparison between long corners and short corners in field hockey. Rawal Med J. 2013;38(4):428-431.

14. Mosquera RP, Molinuevo JS, Román IR. Differences between international men's and women's teams in the strategic action of the penalty corner in field hockey. Int J Perform Anal Sport. 2007;7(3):67-83; doi: 10.1080/ 24748668.2007.11868411.

15. Hopkins WG. Measures of reliability in sports medicine and science. Sports Med. 2000;30(1):1-15; doi: 10.2165/00007256-200030010-00001.

16. Landis JR, Koch GG. The measurement of observer agreement for categorical data. Biometrics. 1977;33(1): 159-174; doi: 10.2307/2529310.

17. Noël B, Furley P, van der Kamp J, Dicks M, Memmert D. The development of a method for identifying penalty kick strategies in association football. J Sports Sci. 2015;33(1):1-10; doi: 10.1080/02640414.2014.926383.

18. Field A. Discovering statistics using SPSS, $3^{\text {rd }}$ ed. London: SAGE Publications; 2009.

19. Laird P, Sutherland P. Penalty corners in field hockey: a guide to success. Int J Perform Anal Sport. 2003;3(1): 19-26; doi: 10.1080/24748668.2003.11868270.

20. Sunderland C, Bussell C, Atkinson G, Alltree R, Kates M. Patterns of play and goals scored in international standard women's field-hockey. Int J Perform Anal Sport. 2006;6(1):13-29; doi: 10.1080/24748668.2006.11868352.

21. López De Subijana C, Juárez D, Mallo J, Navarro E. Biomechanical analysis of the penalty-corner dragflick of elite male and female hockey players. Sports Biomech.2010;9(2):72-78; doi:10.1080/14763141.2010. 495414. 